\title{
BIOMASSAS DE PARTES AÉREAS EM PLANTAS DA CAATINGA ${ }^{1}$
}

\author{
Grécia Cavalcanti Silva², Everardo Valadares de Sa Barretto Sampaio³
}

\begin{abstract}
RESUMO - As biomassas de partes aéreas de nove espécies da caatinga foram determinadas e relacionadas com as medidas das plantas, cortando-se 30 plantas de cada espécie e separando-as em caule, galhos, ramos e folhas. As espécies foram divididas em dois grupos: seis espécies com plantas grandes e três com plantas menores. Cada grupo foi separado em classes de diâmetro do caule (DAP). As alturas totais (HT) dobraram (3,8 a 8,5 m) da classe de menor para a de maior diâmetro (<5 e 27,5-30 cm), as áreas de projeção das copas (APC) aumentaram 14 vezes $\left(4,8\right.$ a $67,3 \mathrm{~m}^{2}$ ) e as biomassas (B) cresceram 113 vezes (4 a $454 \mathrm{~kg}$ ). Os valores máximos foram menores que os de outras formações vegetais tropicais de locais mais úmidos. As proporções das biomassas das partes em relação à biomassa aérea total variaram menos que os valores absolutos, indicando que as plantas vão-se desenvolvendo de forma mais ou menos proporcional. Nas plantas a partir de $17,5 \mathrm{~cm}$ de DAP, cerca de $70 \%$ da biomassa era de caules e galhos maiores que $5 \mathrm{~cm}$ de diâmetro, $20 \%$ de galhos entre 1 e $5 \mathrm{~cm}, 5 \%$ de ramos $<1 \mathrm{~cm}$ e $5 \%$ de folhas. A variável isolada que melhor estimou as biomassas das partes, nos dois grupos de espécies, foi o DAP, com equações de potência $\left(\mathrm{B}=a \mathrm{DAP}^{b}\right)$. Em algumas partes e grupo, HT e APC também foram significativamente correlacionas com as biomassas, embora com $\mathrm{R}^{2}$ inferiores às equações com DAP. Combinando DAP e HT, melhorouse ligeiramente o ajuste, mas não deve compensar o esforço de obter $\mathrm{H}$ no campo. Portanto, as biomassas das partes da planta podem ser estimadas a partir das medidas dos diâmetros do caule, um processo não destrutivo.
\end{abstract}

Palavras-chave: Equação alométrica, folhas e madeira.

\section{ABOVEGROUND BIOMASS OF CAATINGA PLANTS}

\begin{abstract}
Biomass of aboveground parts of nine caating a species were determined and related to plant measurements. Thirty plants of each species were collected and separated into stems, branches, twigs and leaves. The species were divided in two groups: six species of large plants and three species of smaller plants. Each group was divided into classes of stem diameter $(\mathrm{DBH})$. Plant height $(\mathrm{H})$ doubled $(3.8$ to $8.5 \mathrm{~m})$ from the smallestdiameter class to the largest diameter $(<5$ and $27.5-30 \mathrm{~cm})$, canopy projection areas (CPA) increased 14 times $\left(4.8\right.$ to $\left.67.3 \mathrm{~m}^{2}\right)$ and biomass $(B)$ increased 113 times (4 to $454 \mathrm{~kg}$ ). The highest values are below those found in other tropical vegetation types in more humid sites. The ratio of biomass of separated plant parts to total aerial biomass varied less than their absolute values, indicating that plants develop in a relatively uniform way. Plants with DBH above $17.5 \mathrm{~cm}$ had about $70 \%$ of biomass consisting of stems and branches $>5 \mathrm{~cm}$ diameter, $20 \%$ of branches from 1 to $5 \mathrm{~cm}, 5 \%$ of twigs $<1 \mathrm{~cm}$ and $5 \%$ of leaves. DBH was the single variable that best predicted biomass of parts, in both species groups, according to a power equation $\left(B=a D B H^{b}\right)$. $H$ and CPA were also significantly related to biomass for some parts and group, but with $R^{2}$ lower than DBH. Combining DBH and H improved estimation but not enough to justify the extra field effort in determining $H$. Therefore, plant part biomass can be estimated from measurements of stem diameter, in a non-destructive process.
\end{abstract}

Keywords: Allometric equations, leaves and wood.

\footnotetext{
${ }^{1}$ Recebido em 16.03.2007 e aceito para publicação em 19.05.2008.

${ }^{2}$ Departamento de Tecnologia e Ciências Sociais da Universidade do Estado da Bahia (UNEB). E-mail: $\langle$ gcsilva@uneb.br $>$.

${ }^{3}$ Departamento de Energia Nuclear da Universidade Federal Rural de Pernambuco (UFRPE), Recife- PE. E-mail:<esampaio@ ufpe.br>
} 


\section{INTRODUÇÃO}

A vegetação de caatinga recobria originalmente quase todo o semi-árido nordestino, equivalendo a pouco mais da metade dos 1,5 milhão de quilômetros quadrados da região (SAMPAIO, 1995). Atualmente, ainda quase $40 \%$ da área original é recoberta de vegetação nativa. A maior parte dessa vegetação é usada para produção de lenha, seja como finalidade principal, seja como produto da abertura de áreas para plantio no sistema de agricultura itinerante. Outra parte é usada como pastagem nativa, com os animais consumindo a vegetação herbácea presente na época de chuvas e as folhas de árvores e de arbustos que caem ao longo da estação seca (SCHACHT et al., 1989). Apesar desse uso intensivo, pouco se sabe sobre as quantidades de biomassas que são retiradas e recicladas nas partes das plantas utilizadas para esses diversos fins.

Vários trabalhos determinaram os volumes e massas de lenha de algumas áreas de caatinga (CARVALHO etal., 1979; CARVALHOeOLIVEIRA, 1993; CARVALHO e ZÁKIA, 1993; SILVA, 1994), porém não consideraram as biomassas das partes menores. Alguns poucos trabalhos estimaram as biomassas de folhas da caatinga, mas não do resto das plantas (LIMA, 1984). Biomassas totais, sem separação de partes, foram medidas em um único local, em Serra Talhada, PE (KAUFFMAN et al., 1993), e estimadas na região do Seridó, RN (COSTA et al., 2002; AMORIM et al., 2005). Equações para estimar as massas de lenha e as biomassas totais de plantas individuais a partir de medidas como diâmetro de caule e altura têm sido desenvolvidas para algumas espécies e grupos de espécies (ZAKIA et al., 1992; LIMA et al., 1996). Elas têm a vantagem de poder ser usadas em estimativas das biomassas sem derrubada das plantas (NORTHUP et al., 2005).

Um estudo amplo (SILVA, 1998), cobrindo 10 entre as principais espécies da caatinga, foi desenvolvido, no qual foram determinadas equações para estimar biomassas totais e de diferentes partes das plantas. Os resultados das biomassas totais (SAMPAIO e SILVA, 2005 ) indicaram que as plantas podiam ser divididas em três grupos: uma cactácea, três espécies com plantas de porte pequeno e seis espécies de plantas de maior porte. Considerando que a maior parte dos estudos que avaliaram a biomassa de espécies lenhosas da caatinga restringiu a análise à massa seca total da parte aérea, os resultados das medidas das biomassas das partes das plantas, excluindo a cactácea, são apresentados neste trabalho. A cactácea foi excluída por causa de sua estrutura diferente das demais espécies, sem folhas e com ramificações de diâmetros semelhantes. Assim, os objetivos específicos deste trabalho foram determinar as proporções de diferentes partes das plantas em relação à biomassa total da parte aérea e ajustar equações alométricas que permitam estimar as biomassas dessas partes em nove das principais espécies arbustivas e arbóreas da caatinga.

\section{MATERIAL E MÉTODOS}

As nove espécies da caatinga foram selecionadas entre as que têm distribuição mais ampla e maior contribuição para a área basal, em áreas de caatinga, em geral (SAMPAIO, 1996). Elas foram divididas em dois grupos: 1) o primeiro incluiu espécies com plantas de menor porte e foi composto de Aspidosperma pyrifolium Mart., Croton sonderianus Muell. Arg. e Jatropha mollissima (Pohl.) Baill.; e 2) o segundo incluiu arbóreas de maior porte das caatingas e foi composto de Anadenanthera macrocarpa (Benth.) Brenan, Caesalpinia pyramidalis Tul., Maytenus rigida Mart., Mimosa hostilis Benth., Myracrodruon urundeuva Fr. All. e Schinopsis glabra (Engl.) F.A. Barkly \& T. Mey. Para simplificar o texto, o primeiro grupo será denominado "espécies pequenas" e o segundo, "espécies grandes".

Em dois locais de caatinga, foram escolhidas 30 plantas de cada espécie, de forma a representar a faixa usual de tamanho que essas espécies têm na caatinga. Os dois locais situam-se na Depressão Sertaneja, a unidade geoambiental mais típica do semi-árido nordestino,

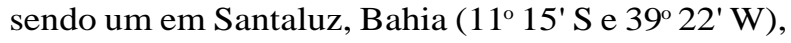
e outro em Petrolina, $\mathrm{PE}\left(9^{\circ} 23^{\prime} \mathrm{S}\right.$ e $\left.40^{\circ} 30^{\prime} \mathrm{W}\right)$. Ambos têm temperatura média em torno de $26^{\circ} \mathrm{C}$, evapotranspiração potencial de 1.500 a $2.000 \mathrm{~mm}$ por ano e precipitações muito variáveis, de ano a ano, com médias anuais de 710 e $430 \mathrm{~mm}$, respectivamente. Os solos dos dois locais são um mosaico de Neossolos Litólicos, Luvissolos e Argissolos Vermelho-Amarelos, de fertilidade baixa a média.

As 30 plantas foram escolhidas com um diâmetro mínimo na base do caule de $3 \mathrm{~cm}$, o padrão para amostragem de caatinga (RODAL et al., 1992), até o máximo encontrado em cada área. A escolha individual foi aleatória, evitando plantas anormais (parcialmente 
cortada, queimada ou tombada) e de forma a ir cobrindo a faixa de tamanho encontrada, com intervalos aproximadamente semelhantes nos tamanhos de diâmetros consecutivos. Cada planta escolhida teve medido seu perímetro do caule à altura do peito $(1,3 \mathrm{~m})$ e ao nível do solo e nos diâmetros ortogonais da projeção da copa e, em seguida, foi tombada sobre uma lona. Os perímetros foram convertidos em diâmetros à altura do peito (DAP) e ao nível do solo (DNS), assumindo uma forma circular, e a área de projeção da copa (APC) foi calculada considerando-se uma forma elíptica. Depois de tombada, a planta teve a sua altura total (HT) e a altura do caule principal (HC) medidos e foi separada em diferentes partes: 1) caule e galhos $>10 \mathrm{~cm}$ de diâmetro; 2) galhos entre 5,1 e $10 \mathrm{~cm}$ de diâmetro; 3) galhos entre 1,1 e $5 \mathrm{~cm}$ de diâmetro; 4) ramos $\leq 1 \mathrm{~cm}$ de diâmetro; e 5) folhas. Como algumas espécies da caatinga são decíduas, as medidas foram feitas na segunda metade da estação das chuvas, quando as copas estavam bem folhadas. As massas de cada parte foram determinadas e amostradas para posterior determinação dos teores de umidade (a $60^{\circ} \mathrm{C}$ ) e de nutrientes.

Para apresentação de alguns dos resultados, as plantas dos dois grupos de espécies foram separadas em classes de diâmetro do caule, iniciando com uma classe daquelas com menos de $5 \mathrm{~cm}$ de DAP e prosseguindo com intervalos de $2,5 \mathrm{~cm}$. As relações entre biomassas e medidas das plantas foram testadas usando-se os valores individuais e diferentes modelos e combinações de variáveis, com o Programa Statistica (STATSOFT, 1995). Os ajustes foram avaliados pelos coeficientes de determinação $\left(\mathrm{R}^{2}\right)$ e pela raiz quadrada do quadrado médio do resíduo. Apenas os modelos e as equações com os melhores ajustes são mostrados.

\section{RESULTADOS E DISCUSSÃO}

Áreas basais, alturas totais e dos caules, áreas de projeção das copas e biomassas foram maiores nas plantas de classes de maiores diâmetros, tanto do grupo das espécies pequenas quanto do grupo das espécies grandes (Tabela 1). Em geral, na mesma classe os valores das espécies pequenas foram menores que os das espécies grandes, exceto na área basal, que é uma variável diretamente relacionada à própria definição da classe. Naturalmente, as variáveis que incorporam dimensões maiores tiveram maiores aumentos; assim, enquanto as alturas totais pouco mais que dobraram da classe de menor para a de maior diâmetro $(3,8$ a 8,5 m), as áreas de projeção das copas aumentaram 14 vezes $(4,8$ a $67,3 \mathrm{~m}^{2}$ ) e as biomassas, 113 vezes ( 4 a $454 \mathrm{~kg}$ ).

Quadro 1 - Área basal (AB, $\mathrm{cm}^{2}$ ), altura total (HT, m), altura do caule principal (HC, m), área de projeção da copa $\left(\mathrm{APC}, \mathrm{m}^{2}\right)$ e biomassa da parte aérea $(\mathrm{B}, \mathrm{kg})$ de dois grupos de espécies da caatinga (plantas de espécies de maior porte - Anadenanthera macrocarpa, Caesalpinia pyramidalis, Maytenus rigida, Mimosa hostilis, Myracrodruon urundeuva e Schinopsis glabra; e plantas de espécies de menor porte-Aspidosperma pyrifolium, Croton sonderianus e Jatropha mollissima), em função do diâmetro do caule à altura do peito (DAP, $\mathrm{cm})$

Table 1 - Basal area $\left(A B, \mathrm{~cm}^{2}\right)$, plant height $(H T, m)$, main stem height $(H C, m)$, crown projection area $\left(A P C, m^{2}\right)$ and aboveground biomass $(B, \mathrm{~kg})$ of two caatinga species groups (species with large plant size - Anadenanthera macrocarpa, Caesalpinia pyramidalis, Maytenus rigida, Mimosa hostilis, Myracrodruon urundeuva e Schinopsis glabra; and species with small plant size - Aspidosperma pyrifolium, Croton sonderianus e Jatropha mollissima), as a function of stem diameter at breast height $(D A P, \mathrm{~cm})$

\begin{tabular}{|c|c|c|c|c|c|c|c|c|c|c|}
\hline \multirow{2}{*}{$\begin{array}{c}\text { Classe de } \\
\text { DAP }\end{array}$} & \multicolumn{4}{|c|}{ Espécies de Maior Porte } & \multicolumn{6}{|c|}{ Espécies de Menor Porte } \\
\hline & $\mathrm{AB}$ & H T & $\mathrm{HC}$ & APC & B & $\mathrm{AB}$ & $\mathrm{H} \mathrm{T}$ & $\mathrm{HC}$ & APC & B \\
\hline$<5$ & 10 & 3,8 & 1,8 & 4,8 & 4 & 11 & 3,4 & 1,4 & 2,6 & 3 \\
\hline $5,1-7,5$ & 32 & 4,8 & 1,4 & 9,5 & 15 & 31 & 4,3 & 1,6 & 6,1 & 8 \\
\hline $7,6-10,0$ & 62 & 5,2 & 1,4 & 19,3 & 33 & 64 & 4,8 & 1,5 & 10,1 & 19 \\
\hline $10,1-12,5$ & 96 & 5,4 & 1,6 & 19,0 & 52 & 99 & 5,2 & 1,4 & 13,1 & 34 \\
\hline $12,6-15,0$ & 143 & 6,6 & 2,3 & 27,9 & 85 & 141 & 5,6 & 1,1 & 18,5 & 42 \\
\hline $15,1-17,5$ & 211 & 7,1 & 2,2 & 30,8 & 120 & 223 & 6,0 & 1,7 & 16,5 & 63 \\
\hline $17,6-20,0$ & 283 & 7,8 & 2,9 & 40,7 & 175 & - & - & - & - & - \\
\hline $20,1-22,5$ & 352 & 8,5 & 3,1 & 42,2 & 230 & - & - & - & - & - \\
\hline $22,6-25,0$ & 424 & 8,4 & 3,3 & 58,8 & 294 & - & - & - & - & - \\
\hline $25,1-27,5$ & 514 & 8,3 & 3,0 & 46,8 & 336 & - & - & - & - & - \\
\hline $27,6-30,0$ & 726 & 8,5 & 4,0 & 67,3 & 454 & - & - & - & - & - \\
\hline
\end{tabular}


Os valores médios das plantas da classe de maiores diâmetros são relativamente baixos quando comparados com os de outras formações vegetais arbóreas tropicais de locais mais úmidos, tanto da própria região (SAMPAIO, 1996) quanto fora dela (KELLER et al., 2001; KETTERINGS et al., 2001; SALIS et al., 2006). No entanto, há que considerar que as plantas escolhidas não cobriram toda a faixa de tamanhos que existem na caatinga, não tendo sido amostradas plantas com diâmetros à altura do peito (DAP) maiores que $30 \mathrm{~cm}$. Plantas maiores existem, embora tenham sido pouco registradas pela carência de trabalhos com essa medida (SAMPAIO, 1996). Há mais trabalhos com medidas de diâmetros na base dos caules, já que foi essa a forma de medida recomendada para a caatinga (RODAL et al., 1992). Como as duas medidas foram fortemente relacionadas (Figura 1), podem ser convertidas sem grande perda de precisão. Diâmetros na base de até $63 \mathrm{~cm}$ (equivalentes a cerca de $50 \mathrm{~cm}$ de DAP) têm sido relatados (PEREIRA et al., 2002), mas são pouco freqüentes, e só proporção pequena das plantas de cada local ultrapassa $27 \mathrm{~cm}$ de diâmetro na base (ALCOFORADO FILHO et al., 2003). Os indivíduos mais altos incluídos na amostragem tinham $12 \mathrm{~m}$ de altura. Há registros de alturas alcançando $19 \mathrm{~m}$ nas áreas de caatingas mais úmidas, mas são poucos os indivíduos que ultrapassam $8 \mathrm{~m}$ (ALCOFORADO FILHO et al., 2003). Comparações dos valores de biomassas e áreas de copas não puderam ser feitas, porque não foram encontrados dados para plantas individuais de espécies da caatinga.

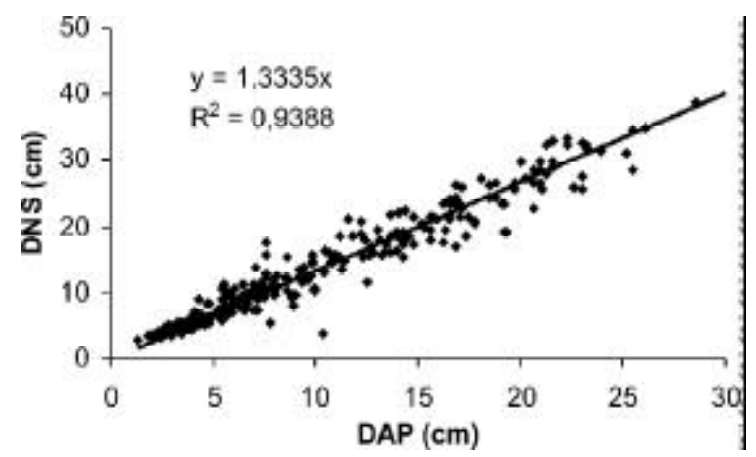

Figura 1 - Relação entre diâmetro do caule à altura do peito (DAP) e ao nível do solo (DNS).

Figure 1-Relationship between stem diameter at breast height (DAP) and at soil level (DNS).

R. Árvore, Viçosa-MG, v.32, n.3, p.567-575, 2008
As proporções das biomassas das partes das plantas em relação à biomassa aérea total (Figuras 2 e 3 ) variaram bem menos que os valores absolutos. Isso indica que as partes das plantas vão se desenvolvendo de forma mais ou menos proporcional (KING, 2005). Logicamente, nas plantas de classes de diâmetros menores as proporções de galhos mais grossos foram menores e até nulas, enquanto as dos galhos finos e folhas foram maiores. Com o aumento dos diâmetros, as proporções foram se invertendo e, nas espécies grandes, atingiram certa estabilidade a partir da classe de 17,5 cm de DAP (Figura 2). Nas espécies pequenas, o equilíbrio não parece ter sido atingido, com proporções crescentes de caules com mais de $10 \mathrm{~cm}$ de diâmetro (Figura 3). Nos dois grupos de espécies, as proporções foram quase as mesmas na mesma classe de diâmetro, reforçando a idéia do crescimento harmonioso das partes, em todas as plantas. A proporcionalidade das partes também pode ser avaliada pela correlação entre suas biomassas. Nesse sentido, merece destaque a alta relação das massas dos ramos $<1 \mathrm{~cm}$ e das folhas, tanto nos dois grupos quanto em todas as plantas $\left(\mathrm{B}_{\text {folhas }}=\right.$ $\left.0,84 \mathrm{~B}_{\text {ramos }} ; \mathrm{R}^{2}=0,95 ; \mathrm{e} \mathrm{p}<0,01\right)$.

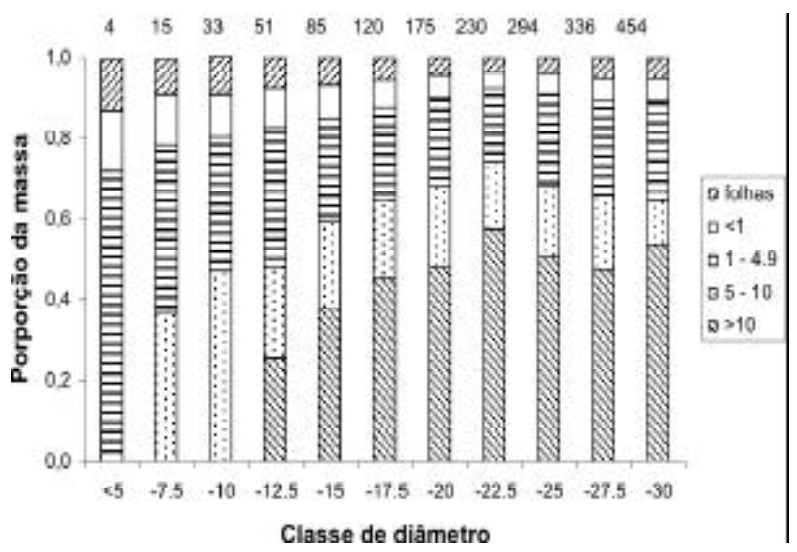

Figura 2 - Proporções da biomassa aérea (números sobre as barras correspondem a biomassas médias em $\mathrm{kg}$ planta-1) em partes da planta (folhas e caules de diferentes diâmetros), por classe de diâmetro do caule, em plantas de seis espécies de maior porte.

Figure 2-Aboveground biomass (numbers above bars correspond to average biomass in $\mathrm{kg}$ plant-1) proportions at plant parts (leaves and branches of different diameters), for classes of stem diameter, for plants of six species with large size. 


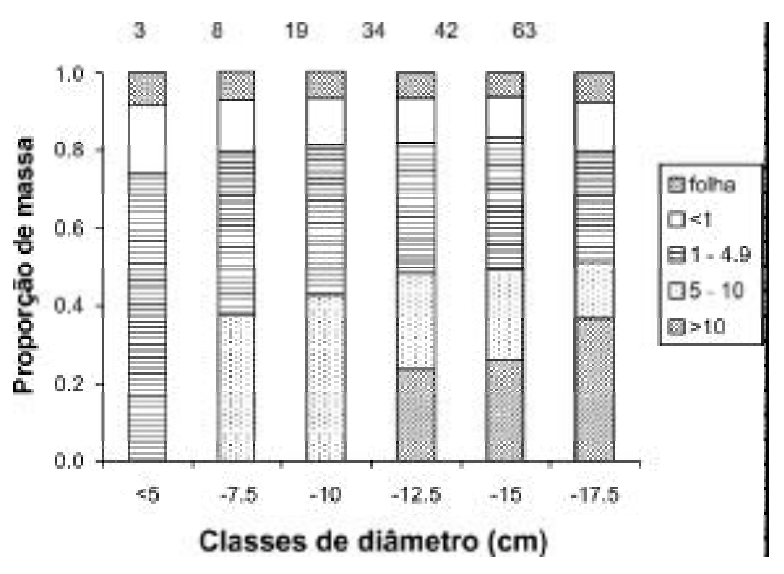

Figura 3 - Proporções da biomassa aérea (números sobre as barras correspondem a biomassas médias em kg planta-1) em partes da planta (folhas e caules de diferentes diâmetros), por classe de diâmetro do caule, em plantas de três espécies de menor porte.

Figure 3-Aboveground biomass (numbers above bars correspond to average biomass in $\mathrm{kg}$ plant-1) proportions at plant parts (leaves and branches of different diameters), for classes of stem diameter, for plants of three species with small size.

Nas espécies grandes, a partir da estabilidade dos $17,5 \mathrm{~cm}$ DAP, cerca de $70 \%$ da biomassa era de caules e galhos maiores que $5 \mathrm{~cm}$ de diâmetro, $20 \%$ de galhos entre 1 e $5 \mathrm{~cm}, 5 \%$ de ramos $<1 \mathrm{~cm}$ e $5 \%$ de folhas.

As proporções dessas duas últimas partes foram sempre relativamente pequenas, não ultrapassando 17 e $13 \%$ nas plantas da classe de menor diâmetro e caindo rapidamente nas classes seguintes. Em outros tipos de vegetação, as proporções das massas de folhas são da mesma ordem de grandeza, chegando a 10-30\% em plantas menores (KUMMAR et al., 1998; KETTERINGS et al., 2001; RAYACHHETRY etal.,2001; RONDON, 2002; OLIVEIRA NETOetal.,2003; NORTHUPetal., 2005; PEICHLe ARAIN, 2007; PAUL et al., 2008) mas ficando, usualmente, na faixa de 2-5\% (BROWN et al., 1995; MOREIRA-BURGER e DELITTI, 1999; REGINA, 2000; SOARES e OLIVEIRA, 2002; SALIS et al., 2006). A comparação das proporções de biomassas nas outras partes das plantas é mais complicada, porque as separações das partes é feita com critérios variáveis e, às vezes, não descritos em detalhe. Em geral, a proporção da biomassa aérea nos caules e galhos mais grossos fica acima de 70\% (BROWN et al., 1995; REGINA, 2000; RAYACHHETRY et al., 2001; RONDON, 2002; SOARES e OLIVEIRA, 2002; SWAMY et al., 2004; SALIS et al., 2006; PEICHL e ARAIN, 2007; PAULetal., 2008), exceto em plantas pequenas (KUMMAR et al., 1998; OLIVEIRA NETO et al., 2003; PAUL et al., 2008). As proporções em ramos mais finos que $3-4 \mathrm{~cm}$ de diâmetro variou de 3 a $15 \%$, com média de 7\% (BROWN et al., 1995), e a de ramos mais finos que $3,2 \mathrm{~cm}$ de diâmetro variaram de 1 a $19 \%$, com média de $8 \%$, enquanto a de galhos entre 3,2 e $6,4 \mathrm{~cm}$ variou de 2 a $49 \%$, com média de 19\% (KETTERINGS et al., 2001). São proporções semelhantes às encontradas nas plantas da caatinga, indicando que o padrão de crescimento geral não varia muito, em diferentes condições de crescimento. As proporções devem ficar contidas em limites estabelecidos pelas possibilidades estruturais do formato das árvores (CHAMBERS et al., 2001; KING, 2005; FEHRMANN e KLEINN, 2006; PILLI et al., 2006).

Dividindo as partes em dois grupos, folhas e ramos $<1 \mathrm{~cm}$, por um lado, e galhos de $1 \mathrm{~cm}$ ou mais, por outro, as proporções variaram pouco, ficando em torno de $10-15 \%$ no primeiro grupo e $85-90 \%$ no segundo. Essa constância facilita a estimativa das biomassas disponíveis nos dois grupos funcionais que corres-pondem a essas partes das plantas. A quase totalidade das folhas e boa parte dos ramos finos são formadas e renovadas anualmente. Folhas e ramos finos são consumidos pelos animais, seja arrancados diretamente das plantas quando elas estão em alturas que os animais alcançam (em torno de $2 \mathrm{~m}$ ), seja apanhados do chão quando caemjásecos(LIMA, 1984; SCHACHTetal., 1989). Osgalhos mais grossos que $1 \mathrm{~cm}$ são mais permanentes nas plantas e usados como lenha.

Aplicando essas proporções ao valor da biomassa total da vegetação da caatinga $\left(74 \mathrm{Mg} \mathrm{ha}^{-1}\right)$, medida em Serra Talhada (KAUFFMANN et al., 1993), as folhas e os ramos finos corresponderiam a cerca de $4 \mathrm{Mg} \mathrm{ha}^{1}$, em cada uma das partes. Com relação aos valores de biomassas totais estimados no Seridó (COSTA etal., 2002), as massas de folhas ou de ramos finos corresponderiam a uma variação de 0,1 a 2,2 $\mathrm{Mg} \mathrm{ha}^{-1}$. Esses valores dentro da faixa calculada para produçãoanual nacaatinga(SCHACHTetal., 1989; SAMPAIO, 1995) e para fitomassa disponível para bovinos em caatinga (LIMA, 1984), também em Serra Talhada, próxima da área amostrada por Kauffmann et al. (1993).

A variável isolada que melhor estimou as biomassas das partes, nos dois grupos de espécies, foi o DAP. Como aconteceu com as estimativas das biomassas totais (SAMPAIO e SILVA, 2005), as equações que melhor se ajustaram aos dados foram do tipo potência: biomassa $=a \mathrm{DAP}^{\mathrm{b}}($ Tabela 2$)$. Em algumas partes e grupo, a altura total (HT) e a área de projeção da copa (APC) também foram significativamente correlacionas com as massas,

R. Árvore, Viçosa-MG, v.32, n.3, p.567-575, 2008 
embora, em geral, com $\mathrm{R}^{2}$ inferiores (dados não mostrados) às equações com DAP. A altura do caule (HC) não foi significativamente relacionada em nenhum caso. Também, não foi o volume das copas (APC x (HT - HC)) com as biomassas de folhas, ramos ou galhos. Das muitas combinações possíveis de duas ou mais variáveis, as de DAP xHT ou ABP(áreabasal daplanta) xHT, quecorresponde ao volume estimado, melhoraram ligeiramente o ajuste das massas no caso das espécies pequenas, mas não no caso das espécies grandes. Nenhuma outra combinação melhorou o ajuste dos dados acima do obtido com o DAP. Em nenhuma das partes, os ajustes foram tão bons quanto nas biomassas totais, provavelmente porque na biomassa total os desvios de uma parte são compensados por desvios de sentido oposto de alguma outra parte. Em geral, os piores ajustes foram nas estimativas de folhas, como tem sido observado em outros trabalhos (HAASEe HAASE, 1995; VEGA et al., 2004; SALIS et al., 2006; BALBOA-MURIAS et al., 2006; PEICHL e ARAIN, 2007). Possivelmente, isso se explica por serem as partes de menor duração nas plantas, ficando mais dependentes de variações na época de formação e tendo sua medida mais influenciada pela época de realização, dentro do curto período de formação e queda na caatinga, além da possível influência de diferenças nas densidades de plantas e no sombreamento (SALIS et al., 2006; BALBOA-MURIAS et al., 2006).

Entre as variáveis usadas, o DAP é a mais facilmente medida, e já há algumas dezenas de trabalhos com informações sobre diâmetros de plantas em áreas de caatinga. Em geral, as medidas têm sido de diâmetro na base, mas, convertendo para DAP (Figura 1) e aplicando as equações obtidas (Tabela 2), poder-se-iam estimar as biomassas das partes das plantas, nessas áreas. A altura é uma medida mais difícil de obter e tem sido informada em um número de trabalhos bem menor do que os que informam os diâmetros. Além disso, na maior parte dos trabalhos em caatinga, tem sido estimada e não medida diretamente. Assim, não há um conjunto de dados que permitam o uso da altura na estimativa de massas de partes das plantas. A combinação de DAP e altura também não é recomendada, porque a melhora na estimativa foi pequena e não justificaria o esforço adicional, no campo, para determinar HT. Vários trabalhos, em outras formações vegetais, têm chegado à conclusão semelhante: pequena melhora no ajuste que não justifica o esforço adicional (HAASE e HAASE, 1995; RAYACHHETRY et al., 2001; SALIS et al., 2006; FEHRMANN e KLEINN, 2006). A medida de projeção das copas também é trabalhosa e exige experiência, tendo sido feita em poucos casos, em áreas de caatinga (ALBUQUERQUE et al., 1982; AMORIM et al., 2005). Em espécies do Pantanal (SALIS et al., 2006), a inclusão da área de copa nas equações melhorou um pouco a estimativa das massas de galhos e folhas, mas esses autores não recomendaram seu uso nas conclusões deste trabalho. Também, não é recomendada para estimativas rotineiras das biomassas das partes das plantas da caatinga. Entretanto, é desejável que mais trabalhos obtenham dados de projeção das copas pelo seu valor como indicadora da cobertura do solo e da ocupação do espaço (AMORIM et al., 2005) e para a confirmação de sua relação com os diâmetros de caule (SAMPAIO e SILVA, 2005).

Quadro 2 - Equações alométricas para calcular a massa seca (kg) de partes das plantas de dois grupos de espécies da caatinga (plantas de espécies de maior porte - Anadenanthera macrocarpa, Caesalpinia pyramidalis, Maytenus rigida, Mimosa hostilis, Myracrodruon urundeuva e Schinopsis glabra; e plantas de espécies de menor porte - Aspidosperma pyrifolium, Croton sonderianus e Jatropha mollissima), em função do diâmetro do caule à altura do peito $(\mathrm{DAP}, \mathrm{cm}) \cdot \mathrm{R}^{2}=$ coeficiente de determinação

Table 2 -Allometric equations to calculate biomass $(\mathrm{kg})$ of plant parts belonging to two caatinga species groups (species with large plant size - Anadenanthera macrocarpa, Caesalpinia pyramidalis, Maytenus rigida, Mimosa hostilis, Myracrodruon urundeuva e Schinopsis glabra; and species with small plant size - Aspidosperma pyrifolium, Croton sonderianus e Jatropha mollissima), as a function of stem diameter at breast height $(D A P, \mathrm{~cm}) . R^{2}=$ coefficient of determinatio.

\begin{tabular}{|c|c|c|c|c|}
\hline \multirow[t]{2}{*}{ Partes da Planta } & \multicolumn{2}{|c|}{ Espécies de Maior Porte } & \multicolumn{2}{|c|}{ Espécies de Menor Porte } \\
\hline & Equação & $\mathrm{R}^{2}$ & Equação & $\mathrm{R}^{2}$ \\
\hline Caule e galhos $>10 \mathrm{~cm}$ & 0,0064 DAP 3,1733 & 0,754 & 0,0082 DAP 2,8151 & 0,606 \\
\hline Caule e galhos 5,1 a $10 \mathrm{~cm}$ & 0,1497 DAP 1,7876 & 0,769 & 0,1331 DAP 1,5784 & 0,554 \\
\hline Caule e galhos 1,1 a $5 \mathrm{~cm}$ & 0,2255 DAP ${ }^{1,7212}$ & 0,852 & 0,5737 DAP 1,6847 & 0,721 \\
\hline Caule e galhos $\leq 1 \mathrm{~cm}$ & 0,0857 DAP 1,5864 & 0,791 & 0,0399 DAP 1,7616 & 0,587 \\
\hline Folhas & 0,0681 DAP ${ }^{1,5829}$ & 0,740 & 0,1900 DAP 2,0515 & 0,648 \\
\hline Parte aérea total & 0,2368 DAP 2,2219 & 0,946 & 0,2627 DAP 1,9010 & 0,819 \\
\hline
\end{tabular}

R. Árvore, Viçosa-MG, v.32, n.3, p.567-575, 2008 


\section{CONCLUSÕES}

As proporções das biomassas das partes de plantas de tamanhos crescentes variaram menos que suas biomassas absolutas, tendendo à estabilização, a partir de $17,5 \mathrm{~cm}$ de DAP, em valores ao redor de $70 \% \mathrm{em}$ caules e galhos maiores que $5 \mathrm{~cm}$ de diâmetro, $20 \%$ em galhos entre 1 e $5 \mathrm{~cm}, 5 \%$ em ramos $<1 \mathrm{~cm}$ e $5 \%$ em folhas. Isso indica que o crescimento das partes é relativamente harmonioso. As biomassas nas partes podem ser estimadas, usando-se equações de potência, a partir dos diâmetros dos caules ao nível do peito ou do solo, que foram altamente correlacionados. Outras variáveis alométricas foram estimadores menos eficientes que os diâmetros.

\section{AGRADECIMENTOS}

Ao CNPq e ao Inter-American Institute for Global Change Research, pelo apoio concedido à pesquisa.

\section{REFERÊNCIAS}

ALBUQUERQUE, S. G.; SOARES, J. G. G.; ARAÚJO FILHO, J. A. Densidade de espécies arbustivas em vegetação de caatinga. Petrolina: EMBRAPA-CPATSA. 1982. 9p. (Pesquisa em andamento, 16).

ALCOFORADO FILHO, F. G.; SAMPAIO, E. V. S. B.; RODAL, M. J. N. 2003. Florística e

fitossociologia de um remanescente de vegetação caducifólia arbórea em Caruaru, Pernambuco.

Acta Botanica Brasilica, v.17, n.2, p.287303, 2003.

AMORIM, I. L.; SAMPAIO, E. V. S. B.; ARAÚJO, E. L. Flora e estrutura da vegetação arbustivoarbórea de uma área de caatinga do Seridó, RN, Brasil. Acta Botanica Brasilica, v.19, n.3, p.615-623, 2005.

BALBOA-MURIAS, M. A.; RODRÍGUEZSOALLEIRO, R.; MERINO, R.; ÁLVAREZGONZÁLEZ, J .G. Temporal variations and distribution of carbon stocks in aboveground biomass of radiata pine and maritime pine pure stands under different silvicultural alternatives. Forest Ecology and Management, v. 237, n.1-3, p. 29-38, 2006.
BROWN, I. F.; MARTINELLI, L. A.; THOMAS, W. W.; MOREIRA, M. Z.; FERREIRA, C. A.;

VICTORIA, R. A. 1995. Uncertainty in the biomass of Amazonia forests: an example from Rondônia,

Brazil. Forest Ecology and Management, v. 75, n.1-3, p. 175-189, 1995.

CARVALHO, A. J. E.; OLIVEIRA, C. R.Avaliação do estoque lenhoso - inventário florestal do estado do Ceará. Fortaleza, Projeto PNUD / FAO / IBAMA / SDU / SEMACE, 1993.61 p.

(Documento de Campo 26).

CARVALHO, A. J. E.; ZÁKIA, M. J .B.

Avaliação do estoque madeireiro: etapa final - inventário florestal do estado do Rio Grande do Norte . Natal, PNUD / FAO / IBAMA / Governo do Rio Grande do Norte, 1993. 85 p. (Documento de Campo 13).

CARVALHO, G. H.; CARVALHO, M. L. R.; LEITE, C. R.; NERI, A. F. O.; CAVALCANTI, J. C.; ALMEIDA, J. C. B. Contribuição para a determinação da potencialidade madeireira da bacia do São Francisco: estado da Bahia. Recife, SUDENE, 1979. 85 p. (Recursos Vegetais 8).

CHAMBERS, J. K.; SANTOS, J.; RIBEIRO, R. J.; HIGUCHI, N. 2001. Tree damage, allometric relationships, and above-ground net primary production in central Amazon forest. Forest Ecology and Management, v. 152, n. 1, p.73-84, 2001.

COSTA, T. C. C.; ACCIOLY, L. J. O.; OLIVEIRA, M. A. J.; BURGOS, N.; SILVA, F. H. B. B. Phytomass mapping of the "Seridó caatinga" vegetation by the plant area and the normalized difference vegetation indeces. Scientia Agricola, v. 59, n. 4, p. 707-715, 2002.

FEHRMANN, L.; KLEINN, C. General considerations about the use of allometric equations for biomass estimations on the example of Norway spruce in Central Europe. Forest Ecology and Management, v. 236, n.2-3, p.412-421, 2006.

HAASE, R.; HAASE, P. Above-ground biomass estimates for invasive trees and shrubs in the Pantanal of Mato Grosso, Brazil. Forest Ecology and Management, v. 73, n. 1, p. 29-35, 1995. 
KAUFFMAN, J. B.; SANFORD JR., R. L.; CUMMINGS, D. L.; SALCEDO, I. H.; SAMPAIO, E. V. S. B. Biomass and nutrient dynamics associated with slash fires in neotropical dry forests. Ecology, v. 74, n. 1, p. 140-151, 1993.

KELLER, M.; PALACE, M.; HURTT, G. Biomass estimation in the Tapajos National Forest, Brazil: examination of sampling and allometric uncertainties. Forest Ecology and Management, v. 154, n. 3, p. 371-382, 2001.

KETTERINGS, Q. M.; COE, R.; NOORDWIJK, M.; AMBAGAU, Y.; PALM, C. A. Reducing uncertainty in the use of allometric biomass equations for predicting above-ground tree biomass in mixed secondary forests. Forest Ecology and Management, v. 146, n. 1-3, p. 199-209, 2001.

KING, D. A. Linking tree form, allocation and growth with an allometrically explicit model. Ecological Modelling, v. 185, n. 1, p.77-91, 2005.

KUMAR, M.; GEORGE, S. J.; JAMALUDHEEN, V.; SURESH, T. K. Comparison of biomass production, tree allometry and nutrient use efficiency of multipurpose trees grown in woodlot and silvopastoral experiments in Kerala, India. Forest Ecology and Management, v. 112, n. 1-2, p. 145-163, 1998.

LIMA, C. G. F. 1984. Determinação da fitomassa disponível ao acesso animal em caatinga pastejada - região de Ouricuri. 1984. 244 f. Dissertação (Mestrado em Botânica) - Universidade Federal Rural de Pernambuco, Recife, 1984.

LIMA, P.C. F.; OLIVEIRA, E. B.; MACHADO, S. A. Equações para estimativa da biomassa de espécies de Prosopis no semi-árido brasileiro. Boletim de Pesquisa Florestal, v. 32/33, n. 1, p. 67-79, 1996.

MOREIRA-BURGER, D.; DELITTI, W. B. C. Fitomassa epigéa da mata ciliar do rio Mogiguaçu, Itapira - SP. Revista Brasileira de Botânica, v. 22, n. 3, p. 429-435, 1999.

NORTHUP, B. K.; ZITZER, S. F.; MCMURTRY, C. R.; BOUTTON, T. W. Aboveground biomass and carbon and nitrogen content of wood species in a subtropical thornscrub parkland. Journal of Arid Environments, v. 62, n. 1, p. 23-43, 2005.
OLIVEIRA NETO, S. N.; REIS, G. G.; REIS, M. G. F.; NEVES, J. C. L. Produção e distribuição de biomassa em Eucalyptus camaldulensis Dehn. em resposta à adubação e ao espaçamento. Revista Árvore, v. 27, n. 1, p. 15-23, 2003.

PAUL, K. I.; JACOBSEN, K.; KOUL, V.; LEPPERT, P.; SMITH, J. Predicting growth and sequestration of carbon by plantations growing in regions of low rainfall in southern Australia. Forest Ecology and Management, v. 254, n.2, p. 205-216, 2008.

PEICHL, M.; ARAIN, M. A. Allometry and partitioning of above- and belowground tree biomass in an age-sequence of white pine forests. Forest Ecology and Management, v. 253, n.1-3, p. 68-80, 2007.

PEREIRA, I. M.; ANDRADE, L. A.; BARBOSA, M. R. V.; SAMPAIO, E. V. S. B. Composição florística e análise fitossociológica do componente lenhoso de um remanescente de caatinga no Agreste Paraíbano. Acta Botanica Brasilica, v. 16, n. 3, p. 357-369, 2002.

PILLI, R.; ANFODILLO, T.; CARRER, M. Towards a functional and simplified allometry for estimating forest biomass. Forest Ecology and Management, v. 237, n.1-3, p. 583-593, 2006.

RAYACHHETRY, M. B.; VAN, T. K.; CENTER, T. D.; LAROCHE, F. Dry weight estimation of the aboveground components of Melaleuca quinquenervia trees in Southern Florida. Forest Ecology and Management, v. 142, n. 1-3, p. 281-290, 2001.

REGINA, I. S. Biomass estimation and nutrient pools in four Qeurcus pyrenaica in Sierra de Gata Mounts, Salamanca, Spain. Forest Ecology and Management, v. 132, n. 2-3, p. 127-141, 2000.

RODAL, M. J. N.; SAMPAIO, E. V. S. B.; FIGUEIREDO, M. A. Manual sobre métodos de estudo florístico e fitossociológico ecossistema caatinga. Brasília, Sociedade Botânica do Brasil, 1992. 24 p.

RONDON, E. V. Produção de biomassa e crescimento de árvores de Schizolobium amazonicum (Huber) Ducke sob diferentes espaçamentos na região da mata. Revista Árvore, v. 26, n. 5, p. 573-576, 2002. 
SALIS, S. M.; ASSIS, M. A.; MATTOS, P. P.; PIÃO, A. C. S. Estimating the aboveground biomass and wood volume of savanna woodlands in Brazil's Pantanal wetlands based on allometric correlations. Forest Ecology and Management, v. 228, n. 1-3, p. 61-68, 2006.

SAMPAIO, E. V. S. B. Overview of the Brazilian caatinga. In: BULLOCK, S. H.; MOONEY, H. A.; MEDINA, E. (Eds.). Seasonally dry tropical forests. Cambridge: Cambridge University Press, 1995. p. 35-63.

SAMPAIO, E. V. S. B. Fitossociologia. In: SAMPAIO, E. V. S. B.; MAYO, S. J.; BARBOSA, M. R. V. (Eds.) Pesquisa botânica nordestina: progresso e perspectivas. Recife: Sociedade Botânica do Brasil, 1996. p. 203-224.

SAMPAIO, E. V. S. B.; SILVA, G. C. Biomass equations for Brazilian semiarid caatinga plants.Acta

Botanica Brasilica, v. 19, n. 4, p. 937-945, 2005.

SCHACHT, W. H.; MESQUITA, R. C. M.; MALECHEK, J. C.; KIRMSE, R. D. Response of caatinga vegetation to decreasing levels of canopy cover. Pesquisa Agropecuária Brasileira, v. 24, n. 11, p. 1421-1426, 1989.

SILVA, G. C. Relações alométricas de dez espécies vegetais e estimativas de biomassas aéreas da caatinga . 1998. $163 \mathrm{f}$. Tese (Doutorado em Botânica) - Universidade Federal Rural de Pernambuco, Recife. 1998.
SILVA, J. A. Avaliação do estoque lenhoso - inventário florestal do estado da Paraíba. João Pessoa: Projeto PNUD / FAO / IBAMA - Governo da Paraíba, 1994. 27 p. (Documento de Campo 21).

SOARES, C. P. B.; OLIVEIRA, M. L. R. Equações para estimar a quantidade de carbono na parte aérea de árvores de eucalipto em Viçosa, Minas Gerais.

Revista Árvore, v. 26, n. 5, p. 533-539, 2002.

STATSOFT. Statistica for windows, version 5.0. Copyright Statsoft. 1995.

SWAMY, S. L.; KUSHWAHA, S. K.; PURI, S. Tree growth, biomass, allometry and nutrient distribution in Gmelina arborea stands grown in red lateritic soils of Central India. Biomass and Energy, v. 26, n. 4, p. 305-317, 2004.

VEGA, F. V. A.; BOVI, M. L. A.; SPIERING, S. H.; GODOY JÚNIOR, G. Relações alométricas para estimativa da fitomassa aérea da pupunheira. Horticultura Brasileira, v.22, n.1, p.104-108, 2004.

ZÁKIA, M. J. B.; PAREYN, F. G.; RIEGELHAUPT, E. Equações de peso e de volume para oito espécies lenhosa nativas do Seridó, RN. In: IBAMA. Plano de manejo florestal para a região do Seridó do Rio Grande do Norte. Natal: PNUD / FAO / IB AMA - Governo do Rio Grande do Norte, 1992. p. 1-92. 